\title{
Optimal backwashing in dead-end bacterial microfiltration with irreversible attachment mediated by extracellular polymeric substances production
}

\author{
N. G. Cogan $^{1} \quad$ Jian $\mathrm{Li}^{2}$ \\ Appala Raju Badireddy ${ }^{3}$ Shankararaman Chellam ${ }^{4}$
}

${ }^{1}$ Corresponding author. Address: Department of Mathematics, Florida State University, 1017 Academic Way, Tallahassee, Florida 32306, U.S.A., Tel.: (850)6442202, Fax: (850)644-4053

${ }^{2}$ Department of Mathematics, Florida State University, 1017 Academic Way, Tallahassee, Florida 32306, U.S.A

${ }^{3}$ University of Vermont, Burlington, VT, USA, e-mail: raju.badireddy@uvm.edu

${ }^{4}$ Department of Civil Engineering, Texas A \& M University, College Station, TX 77843-3136,e-mail: chellam@tamu.edu 


\begin{abstract}
Microfiltration and ultrafiltration are methods of removing colloidal impurities from water and wastewater. One of the major issues when dealing with the practical implementation of membranes is the reduction of water productivity as the foulants accumulate on the membrane surface or within the pores. Membrane regeneration by periodic backwashing is an effective method of reducing fouling; however, to date the timing and duration of the backwashing for effective fouling control is largely only empirically determined.

In this manuscript, we present an optimal control formulation to determine the timing and duration for membrane regeneration by backwashing. In this formulation, we use the direction of the flow as the control variable and make predictions regarding the optimal protocol. We explicitly include irreversible attachment due to bacterial deposition and biofilm formation on the membrane and demonstrate that irreversible attachment of bacteria has important ramifications for the effective timing of hydraulic backwashes as well as the efficiency in producing clean water. In particular, we find that irreversible attachment and additional fouling due to exo-polymeric substance (EPS) production and biofilm formation decreases the maximum filtration volume. Additionally, as the effectiveness of membrane regeneration declines, the timing of the cycling is also altered. In general, including the role of EPS in biofouling substantially changes predictions of backwashing timing and implies important considerations for practical predictions.
\end{abstract}

Key words: Biofouling, Mathematical modeling, Optimal control, Biofilm, Fouling control 


\section{Introduction}

Dead-end microfiltration (MF) and ultrafiltration (UF) are used in a variety of industrial and municipal settings as efficient and practical methods for removing turbidity, microorganisms, and other colloidal pollutants (1-3). During bacterial filtration, accumulation of microorganisms on the $\mathrm{MF} / \mathrm{UF}$ membrane surface or within its porous matrix causes fouling $(4,5)$. In most environmental applications, the flow is periodically reversed, in the time frame of tens of minutes, to control what is operationally termed "reversible fouling" $(6,7)$. This procedure, variably referred to as backwashing, backflushing, or backpulsing removes a fraction of the accumulated foulant thereby partially regenerating the filter in the short-term (8-12). The complementary portion of foulants that is not removed by backwashing contributes to irreversible fouling in the long-term (13-15). Such foulants are removed by chemical cleaning when productivity drops below a predetermined acceptable level, which is performed typically over a time frame of weeks or months. In practice, the timing and duration of the backwashing cycles is determined empirically - often using proprietary scheduling algorithms developed by the manufacturer. Input is also provided by consulting engineers when on-site pilot-scale testing is performed in support of plant design or to comply with regulations (1).

Since the permeate water is used to flush the filter, frequent backwashing will decrease the net yield of filtered water. Therefore, one aim is to backwash as little as possible. However, when microfiltering bacteria, there is an additional difficulty if the backwashing cycles are too far apart, namely, the formation of a thick cake of irreversibly attached microorganisms on the filter in the form of a biofilm $(16,17)$. Although earlier work has demonstrated the effectiveness of backwashing to control fouling during filtration of colloids, bacteria, and natural water e.g. $(6,7,12,15)$, in general, there is a lesser degree of understanding regarding the development, prevention, and optimal removal of biofilms formed on MF/UF membranes (18). The aim of this manuscript is to extend our previous results to rigorously include the effects of biofilm formation on modeling the dynamics and optimal control of the filtration/regeneration process from a more basic standpoint.

Extracellular polymeric substances (EPS) fouling of membranes is a substantially complex phenomenon since flux decline is dependent on (i) the total concentration, conformation, and composition of each of its individual components including polysaccharides, proteins, lipids as well as nucleic, 
humic, and uronic acids, (ii) its spatial distribution within a multi-species biofilm, (iii) its interactions with other foulants present in the feed water, including microorganisms, and (iv) its propensity and ability to allow bacterial colonization of membrane surfaces $(5,16,18,19)$. EPS fouling mechanisms elucidated using simplistic model solutions $(20,21)$ may not apply to fouling in the simultaneous presence of other non-EPS foulants and in a complex EPS mixture due to differences in the cake layer morphology/permeability caused by variations in foulant transport and interactions with membrane surfaces (22). Similarly, insights gained from biofouling experiments with single bacterial species $(16,23)$ also cannot be easily extrapolated to a more realistic scenario of fouling caused by multiple bacterial species due to differences in the rate of EPS secretion, the composition of EPS, and consequently its specific interactions with the membrane surface.

The main determining factor for the development of irreversibly attached bacteria is the formation of a biofilm. Biofilms consists of microorganisms enmeshed in EPS along with other particulate matter within the bulk water $(24,25)$. EPS facilitates cohesion and adhesion, hydration, and protection of the embedded microbes from predators. Biofilm dynamics have been widely studied because of their impact in disease transmission, corrosive properties, and most importantly their tolerance to disinfection (26). These investigations have shown that it is very difficult to remove an established biofilm using physical or chemical means. Typically, the biofilm typically grows back in a short time once the challenge to the bacteria (antibiotics, biocides, or mechanical scraping) is stopped. Therefore it is vital to prevent the biofilm formation rather than try and treat the established biofilm (18). Consequently, we have proposed to time backwashes before 'significant amounts' of EPS are secreted and bacteria are irreversibly attached to membrane surfaces $(16,17,27,28)$. More importantly, we show here that the timing can be estimated using an optimal control formulation.

We note that mathematical treatment of membrane fouling has a relatively long history (29). One frequent approach consists of empirically driven, blocking-laws that describes the dynamics of the volume of filtered water as the flux or pressure changes $(30,31)$. The goals of these models are to estimate the power law relationship based on experimental observations and thus to diagnose the fouling regime. This is useful in industrial and municipal settings by troubleshooting fouling and helping point to intuitive changes in the filtration regime. However, these models cannot typically be used to predict the fouling behavior for a wider range of settings and are not sim- 
ple to use for mathematical optimization. Recently, we introduced a more rigorous model that describes the change in flux with respect to time, accounting for the reduced flux due to foulant accumulation during constant pressure filtration (32). This model can also be adjusted to describe the change in the pressure during constant flux operation (27). The former is more often used in laboratory/bench models while the latter is more often used in practical applications. Using optimal control theory, we were able to propose the backwashing protocol that optimizes the total volume of clean water which is the main control target used in industry (28). This model captures the experimental data quite well; however, we neglected irreversible attachment, assuming that backwashing was able to remove all of the accumulated foulant, regardless of its residence time on the membrane. In the present study, we demonstrate the effect of irreversible attachment on the optimal control analysis of our model.

Previously, we described the fouling and regeneration process as a twostep process, controlled by a control variable $u$ which denotes the direction of the flow. The flow changes direction during backpulsing so $u=u(t)$ is an explicit function of time (28). Forward filtration is represented by $u=1$, while flow reversal was described by $u=-1$. In constant pressure operation, accumulation occurs during forward filtration at a rate proportional to the flux, which is proportional to the transmembrane pressure drop. The proportionality parameter is the inverse of resistances (resistance in series model) which is related to the foulant on the filter. In regeneration, we assumed that foulant was removed from the filter at a rate proportional to the flux, and that this proportionality was constant. Constant flux operation is represented similarly, with the pressure related to the flux by inverting the resistance in series relation. Here extend our analysis to include irreversible attachment of bacteria by way of EPS formation. EPS affects the system in two primary ways. First, accumulation of EPS on the membrane filter reduces the flux of fluid through the filter by increasing the total resistance to water transport. Second, EPS immobilizes the bacteria and other foulants onto the filter, hindering detachment during flow reversal. Therefore, we also need a description of EPS production. In the next section, we describe the experimental protocol that we use to determine the EPS dynamics that are additional in this study. We then introduce our mathematical model and the framework of optimal control. Following that we describe the implications of EPS formation. We compare our optimal control trajectories with those where regeneration is independent of EPS. When the EPS affects the flux 
(which it almost surely does) the optimal solution is similarly distinct. Finally, we close the manuscript with some remarks regarding directions that remain to be explored.

\section{Mathematical model}

Based on our previous study (28) the filtration and regeneration processes are initially described separately. During the forward filtration process, the accumulation of bacteria, $B$ is proportional to the raw water flux through the membrane, $J$ :

$$
\frac{d B}{d t}=K J .
$$

where $K$ is a constant that describes the water quality. Very high $K$ indicates rapid fouling, e.g. induced by poor water quality.

If we assume the filter is initially clear of bacterial foulants, we have an initial condition:

$$
B(0)=0,
$$

The flux, $J$, is assumed to be proportional to the pressure differential $\Delta P$ and inversely proportional to the resistance on the membrane. This is similar to the empirical descriptions developed by others (33). Here, three kinds of resistance are considered: $R_{m}$, the resistance generated by clean membrane; $R_{b}$, the resistance generated by bacteria; and $R_{e}$ resistance from EPS (denoted $E$ ). The flux is:

$$
J=\frac{\Delta P}{\mu\left(R_{m}+R_{b}+R_{e}\right)},
$$

where $\mu$ is absolute viscosity of the solution that scales the flux/pressure gradient relationship. The clean membrane resistance, $R_{m}$ is assumed to be a constant describing the intrinsic membrane resistance, while the the resistances due to bacteria and EPS are assumed to be proportional to the amount of bacteria and EPS on the filter, respectively,

$$
\begin{aligned}
& R_{b}=\mu_{1} B \\
& R_{e}=\mu_{2} E .
\end{aligned}
$$


Biofilms are formed by almost any bacteria that binds to a surface and begins to produce EPS and become irreversibly attached. In order to account for EPS production but maintain a tractable model, we take the simplest possible model for EPS production where EPS is produced at a rate that is proportional to the density of bound bacteria on the surface while there is simultaneous degradation or decay of EPS:

$$
\begin{aligned}
\frac{d E}{d t} & =m B-\delta E \\
E(0) & =0
\end{aligned}
$$

where $m$ represents the EPS increasing rate and $\delta$ is the EPS degradation rate and we have again assumed the membrane is initially clear of foulants. We note that this is a nutrient-independent model that is consistent with other formulations of EPS dynamical models (34).

In the backwashing process, accumulated foulant on the filter is removed at a rate proportional to the flux through the membrane:

$$
\frac{d B}{d t}=-\hat{K} J B .
$$

The initial condition for this equation depends on how long the filter has been operated in forward mode. That is, we regenerate only when there has been some accumulation of foulant on the filter.

In our previous study (28), the proportionality relation, $\hat{K}$, was assumed to be constant. To describe the impact of EPS formation, here we assume that $\hat{K}$ is a decreasing function of $E$ :

$$
\hat{K}=\frac{1}{c+\alpha E},
$$

Where $c$ is a regularization parameter that scales the regeneration rate of the filter in the absence of EPS and $\alpha$ is a scaling factor governing the rate of decrease in $\hat{K}$. This change substantially alters the analysis. Since the backwashing timing depends explicitly on EPS which in turn depends on the bacterial accumulation, the backwashing depends on the duration of the forward cycle. This implicit dependence between the forward and backwashing cycle makes the analysis more complicated.

It is important to note that we are assuming that the feed water contains only a single species of bacteria - this is clearly an oversimplification; however, this is quite typical in biofilm models. It is relatively straightforward 
to include the affects of multiple species by compartmentalizing the bacteria into separate species (35). This would allow for more detailed modeling of the growth kinetics which are intimately coupled with the EPS production.

\section{Materials and Methods}

Previously, we described how we estimated parameters associated with foulant accumulation, fouling efficiency and membrane resistances. To include the effect of EPS, we need additional parameter estimation. In particular, we require estimates for the kinetics of EPS production, $m$ and $\delta$. EPS affects the system through the membrane resistance, $R_{e}$, which requires an estimate of $\mu_{2}$. We base our estimates on previous experiments (16).

\subsection{Experimental Work}

\section{Bacterial growth, EPS extraction, protein and polysaccharide mea- surements:}

Planktonic cultures ofBrevundimonas diminuta (American Type Culture Collection (ATCC) 19146) was freshly grown aerobically at $30^{\circ} \mathrm{C}$ before each experiment in nutrient broth (Difco). This organism was chosen since it is employed to evaluate the retention characteristics of membranes rated at $0.2 \mu \mathrm{m}$ (36). After 36 hours (approximately at end of exponential phase), the suspension was centrifuged (5000 $\mathrm{g}$ for $15 \mathrm{~min}$ at $4{ }^{\circ} \mathrm{C}$ ) to collect the bacterial pellet, which was washed twice with phosphate-buffered saline solution (PBS). Bacteria were resuspended in PBS at a concentration $\sim 10^{8}$ $\mathrm{CFU} / \mathrm{mL}$.

EPS was extracted using methods reported in the literature e.g.(16, 24, 37). First, free-EPS and any residual growth media were separated from bacterial cells by centrifuging $60 \mathrm{~mL}$ of culture $\left(5000 \mathrm{~g}, 15 \mathrm{~min}, 4^{\circ} \mathrm{C}\right)$. Next bound-EPS was extracted by (i) resuspending and washing the pellet formed in the first step using isotonic buffer $(10 \mathrm{mM}$ Tris/ $\mathrm{HCl} \mathrm{pH} \mathrm{8.0,10} \mathrm{mM} \mathrm{EDTA,}$ $2.5 \% \mathrm{NaCl}$ ), (ii) employing another centrifugation step $\left(5000 \mathrm{~g}, 15 \mathrm{~min}, 4^{\circ} \mathrm{C}\right)$ for further cell purification, (iii) again resuspending the bacterial pellet in buffer, incubating it overnight at $4^{\circ} \mathrm{C}$, and then vigorously vortexing for 5 minutes, and (iv) recovering EPS bound to cell surfaces as the supernatant by centrifugation. Any residual bacterial debris was further separated from 
supernatant solutions representing free-EPS and bound-EPS by centrifuging (12000g, $30 \mathrm{~min}, 4^{\circ} \mathrm{C}$ ) and precipitating with 3 volumes of ice-cold $99.5 \%$ ethanol and incubation at $-20^{\circ} \mathrm{C}$ for $\sim 18 \mathrm{~h}$. Following incubation, suspensions were again centrifuged $\left(12000 \mathrm{~g}, 30 \mathrm{~min}, 4^{\circ} \mathrm{C}\right)$ to recover the precipitated "free" and "bound" EPS from suspensions. Solutions were dialyzed for $72 \mathrm{~h}$ against three changes of nanopure water per day and stored at $20 \mathrm{C}$ before analysis. The modified Lowry assay (Pierce Biotechnology) was used to measure total proteins. Absorbance at $750 \mathrm{~nm}$ was measured using a UV-VIS spectrometer (DR4000, Hach) after calibration with bovine serum albumin (BSA) standards. Total polysaccharides were also measured colorimetrically using the phenol-sulfuric acid method against glucose standards. Results shown in this manuscript correspond to three replicates.

\section{Atomic Force Microscopy:}

An atomic force microscope (Nanoscope IV, Digital Instruments, Santa Barbara, CA) was used to visualize EPS production (see Figure 1). $100 \mu \mathrm{L}$ samples containing bacteria and growth medium were deposited on the surface of an atomically smooth substrate (highly ordered pyrolytic graphite) and air-dried. Images were obtained in the tapping mode under ambient conditions in air using oxide-sharpened microfabricated Si3N4 cantilevers obtained from Park Scientific Instruments (Mountain View, CA, USA) and analyzed using WSxM v4.0 Beta 8.2 (38).

\section{Microfiltration:}

The resistance of the clean membrane was measured first using PBS in the range $3-15$ psig $(21-103 \mathrm{kPa})$. Next, bacteria harvested during early stationary phase, suspended in PBS were microfiltered in the unstirred deadend mode using a $4.1 \mathrm{~cm}^{2}$ effective area commercial cell (model 8010, Millipore). $0.2 \mu \mathrm{m}$ track-etched membranes were employed because they possess a narrow pore size distribution and will retain $B$. diminuta on their surface by definition, thereby restricting internal pore fouling. After microfiltering approximately $10^{11} \mathrm{CFU} / \mathrm{m}^{2}$ the membrane was removed from the cell and kept hydrated in Petri dishes by periodically adding a few drops of nanopure water. After predetermined time intervals (i.e. 0, 1, 2, and 5 days), the filter was removed from the Petri dish and rinsed with $\sim 25 \mathrm{~mL}$ of nanopure water in a squeeze bottle to mimic backwashing $(5,16)$. After the simulated 
backwashes, the membrane resistance was again determined using PBS in the same pressure range (i.e. $21-103 \mathrm{kPa}$ ). Note that the extended duration of bacterial contact with the membrane allowed the establishment of a stronger attachement between EPS production and backwashing.

Parameter Estimation:

To obtain estimates of the kinetic parameters $m$ and $\delta$, we use a simple model of bacterial growth in conjunction with our EPS model. Specifically, we assume that the bacterial dynamics in the experiments described in this section are well described by Michaelis-Menten kinetics (39). At the same time, EPS is produced according to Equation 6. The governing equations are,

$$
\begin{aligned}
\frac{d B}{d t} & =\mu \frac{S}{k_{s}+S} B, \\
\frac{d E}{d t} & =m B-\delta E, \\
\frac{d S}{d t} & =-\frac{\mu}{Y} \frac{S}{k_{s}+S} B,
\end{aligned}
$$

where $S, k_{s}, Y$, and $\mu$ denote the substrate concentration, half-saturation coefficient, yield, and maximal growth rate, respectively.

Using Matlab's fminsearch, we perform an optimization procedure to obtain a best fit estimate for the free parameters. The final comparison between the growth curves and the EPS densities are shown in Figure 2. Note that we are using optical density (OD) as a dimensionless measure of bacterial density. Therefore, we can scale the EPS production for the filtration process. It is emphasized that Figure 2 depicts EPS production by planktonic bacteria. Hence, data shown in Figure 2 should be cautiously extrapolated to sessile biofilms such as those formed on membrane surfaces.

We note that our fit is reasonably accurate, but even more importantly the qualitative predictions (e.g. the relationships between timing for the predictions estimated below) do not depend on precise estimates. Instead, we are finding reasonable estimates for the parameters based on these fits.

The final two parameters that need attention are $c$ and $\alpha$. The former is assumed to yield the regeneration efficiency in the absence of EPS so that $\frac{1}{c}=\hat{K}$ in (28), giving an estimate of $c=100 \mathrm{Lm}^{-2}$. We do not have data to support the specific value of $\alpha$. Here we pick a value that is consistent with 
the time-scale of the decay in removal due to irreversible attachment, but do not claim that this is quantitatively exact - rather, we only claim that the general trend is correct.

\section{Optimal Control Analysis}

The main idea in optimal control is to determine the value of a control function that maximize or minimizes a target function. Optimal control is often thought of as an extension of the standard optimization of a function by determining where the derivative is zero. In fact, the method that we use is very similar to constrained optimization where the goal is to maximize or minimize a function subject to a constraint on the independent variable. These problems are typically solved using Lagrange multipliers where the geometric relationship between the function that is being optimized and the constraint is determined (in particular, the gradients point in the same direction). Here the constraints are the differential equations and the goal is to maximize the Lagrangian (the total volume filtered) but the methods that we use are similar in spirit.

To develop an optimal control problem, we introduce a control factor to $u$ describe the switching from forward filtration to backwashing. Forward filtration is represented by $u=1$ while backwashing is indicated by $u=-1$. In this way, Equations 1 and 7 can be combined:

$$
\frac{d B}{d t}=\frac{(1+u)}{2} K J-\frac{(1-u)}{2} \hat{K} J B
$$

In our previous study we were able to manage the optimal control problem essentially analytically (28) . Here, even though we can formulate the statements implicitly, there does not seem to be a generic way to find the explicit solution. Therefore, we will state the general case and in three sub-cases describe the particular forms and solutions.

The goal of this analysis is to determine the time course of forward filtration and membrane regeneration by determining the $u(t)$ that maximizes the volume of filtered water over the course of filtration. We note that for a physical solution, $u$ must be a piecewise constant. Since clean water is used to regenerate the membrane, we need to determine the total water that is fed through the membrane during forward filtration (when $u=1$ ) minus the water fed through the membrane in the opposite direction during regenera- 
tion (when $u=-1$ ). During the time period $\left[0, T_{\text {total }}\right]$, the total amount of clean water obtained is:

$$
L=\int_{0}^{T_{\text {total }}} u(t) J(t) d t,
$$

since $u$ controls the direction of the flow. We are assuming a unit area for the membrane, and using unit scaling for the flux.

By Pontryagin's maximum principle, if $u^{*}(t)$ and $B^{*}(t)$ denote the optimal solutions, then there is exists a piecewise differentiable adjoint function $\lambda(t)$ such that the Hamiltionian satisfies the optimality condition, $H\left(t, B^{*}(t), u(t), \lambda(t)\right) \leq$ $H\left(t, B^{*}(t), u^{*}(t), \lambda(t)\right)$.

In our case, the Hamiltonian is defined as

$$
\begin{aligned}
H & =u J+\lambda\left(\frac{(1+u)}{2} K J-\frac{(1-u)}{2} \hat{K} J B\right) \\
& =\left(J+\frac{\lambda J}{2}(K+\hat{K} B)\right) u+\frac{\lambda J}{2}(K-\hat{K} B) \\
& =u \Omega+\frac{\lambda J}{2}(K-\hat{K} B)
\end{aligned}
$$

where

$$
\Omega=J+\frac{\lambda J}{2}\left(K+\frac{B}{c+\alpha E}\right)
$$

Pontryagin's principle argues that at the optimal control value, $u^{*}$,

$$
\frac{\partial H}{\partial u}=\Omega=0 .
$$

Since the Hamiltonian is linear control, we refer to this optimal control problem as a bang-bang control with singular state (that is, it is possible that $u^{*}$ is not one of the physically allowable values of the control function) (40). We can define the optimal control as,

$$
u^{*}=\left\{\begin{array}{cc}
1 & \text { if } \Omega>0 \\
-1 & \text { if } \Omega<0 \\
\text { unknown } & \text { if } \Omega=0
\end{array}\right.
$$

the switches coincide with the place where $\Omega$ switches signs (i.e. at singular 
state). According to the Hamiltonian, we have,

$$
\begin{aligned}
& \frac{d B}{d t}=\frac{\partial H}{\partial \lambda} \\
& \frac{d \lambda}{d t}=-\frac{\partial H}{\partial B}
\end{aligned}
$$

The details of the switch timing, and filtration behavior depend on the solution to the dynamic equations. Intuitively, if backwashing is done infrequently there is a build-up of irreversible attachment but if it is done too frequently the clean water is used in excess. The basic idea is to begin with a clean filter and begin forward cycling (i.e. $u^{*}=1$ ) until the system is guided to $\Omega=0$. At that point, if the control were continuous, one would keep the system at the optimal control value. Since $u^{*}$ is required to be either 1 or -1 (based on the filtration design), we have to do something different. By alternating between forward cycling and backwashing at the appropriate timing and duration, we are able to keep the system near $u^{*}$ on average. As the number of cycles increases in a fixed period of time, we get closer and closer to the optimal solution. We note that we have used exactly this methodology in the absence of EPS dynamics previously (28). We refer to this as approximation with a bang-bang control.

Therefore, we must first determine the theoretical optimal control value $u^{*}$, then we decompose the total filtration time, $T$ into $n$ sub-intervals where a periodic cycling is performed. The cycling time, $T_{n}$ is the sum of the forward and backwards cycling: $T_{n}=T_{\text {forward }}+T_{\text {backward }}$. During each of the cycles we keep the average behavior equal to the control value:

$$
u^{*}=\frac{T_{\text {forward }}-T_{\text {backward }}}{T_{n}}
$$

Note that the left side of this is equal to 1 if the filter is run constantly in forward operation, and -1 if it is constantly regenerating the filter. Additionally $0 \leq u^{*} \leq 1$ as required.

To determine the singular control value $u^{*}$, we split our analysis into several simplifying cases. We begin by neglecting the effect of EPS on the flux and removal constant. We then consider the case when the removal constant alone depends on EPS and finally allow both the removal constant and the flux to depend on EPS. We note that we use Matlab's ODE45 suite that employs a fourth order Runga-Kutta method to solve the ODE's numerically. 


\section{Case 1: Effects of neglecting EPS on the flux and $\hat{K}$}

This case is equivalent to the case that we considered in our previous study, but we state it here for comparison with the other cases.

We set $\mu_{2}=0$ in Equation 5 which simplifies the flux,

$$
J=\frac{\Delta P}{\left(\mu R_{m}+\mu_{1} R_{b}\right)} .
$$

Additionally, we set $\alpha=0$ in Equation 8, so that $\hat{K}$ is a constant. In this case, the switching curve, $\Omega$ is given by:

$$
\Omega=\frac{K+B \hat{K}}{2} \lambda+1,
$$

where $\lambda$ is the solution to the adjoint equation.

It is simple to see that $u^{*}=\frac{B \hat{K}-K}{B \hat{K}+K}$ (since we require $B$ to be at a steadystate in order to remain on the switching curve), although we do not have an explicit expression for the solution, $B$. For the parameter values in Table $1, u^{*} \approx 0.16$. In Figure 3 we show the total volume filtered as a function of time, for various bang-bang approximations to the optimal control (with optimal solution shown for comparison). As the frequency of alternating increases, the total volume that is filtered approaches the optimal.

\section{Case 2: $\hat{K}$ depends on EPS}

Here we assume that $\alpha$ is not zero while $\mu_{2}=0$. Therefore the EPS only alters the reversion, but not the flux. We find that the optimal value of $u$ is

$$
u^{*}=\frac{(-\alpha E K-K c+B)}{(\alpha E K+K c+B)} .
$$

Again, we see that $-1 \leq u^{*} \leq 1$ so we will approximate this with a bang-bang approach. From the parameters here, we find that $u^{*} \approx 0.17$. In Figure 4 we show the filtered volume curves.In Case 1 the total volume filtered after 1 hours is $0.83 \mathrm{~L} / \mathrm{m}^{2}$ while for Case 2 it is $0.35 \mathrm{~L} / \mathrm{m}^{2}$, which is a modest decline. This is different than in Case 3 where we also consider the effect of the EPS on the fouling process. 


\section{Case 3: $R_{e}$ and $\hat{K}$ depend on EPS}

The optimal value of the control parameter $u$ is the same as in Case 2 . However, the value that we obtain after determining the values of $B(t)$ and $E(t)$ is quite different: $u^{*} \approx 0.31$ indicating that we need to refrain from reversing the flow for a bit longer than when fouling due to EPS is neglected. However, there is a clear reduction in the volume filtered due to the presence of the EPS. The total volume that is filtered in the 1 hours is $0.22 \mathrm{~L} / \mathrm{m}^{2}$.

It is notable that the curves that approach the optimal value do so at different 'rates' (meaning as the number of cycles increase) fro each of the three cases. This implies that as the effect of EPS is included it becomes more difficult to attain the theoretical optimal. In order to achieve the theoretical

\begin{tabular}{|c|c|c|}
\hline \hline Variable & Description (Units) & Value \\
\hline$B$ & Foulant density on the filter $\left(g L^{-1}\right)$ & Variable \\
$E$ & EPS density on the filter $\left(g L^{-1}\right)$ & Variable \\
$J$ & Flux $\left(\mathrm{Lm}^{-2} \mathrm{hr}^{-1}\right)$ & Variable \\
$R_{m, b, e}$ & Membrane, Foulant, EPS, Resistance (resp.) $\left(\mathrm{m}^{-1}\right)$ & Variable \\
$u$ & Flow direction, control (dimensionless) & $1,-1$ \\
\hline \hline Parameter & Description (Units) & Value \\
\hline$\Delta P$ & Pressure drop (Psig) & 2 \\
$\mu$ & Absolute viscosity $\left(N \mathrm{Nm}^{-2}\right)$ & $10^{-3}$ \\
$R_{m}$ & Membrane resistance $\left(\mathrm{m}^{-1}\right)$ & $10^{-7}$ \\
$\mu_{1}$ & Foulant efficiency $\left(L g^{-1} m^{-1}\right)$ & $10^{-5}$ \\
$\mu_{2}$ & EPS efficiency $\left(L g^{-1} m^{-1}\right)$ & $10^{-7}$ \\
$K$ & Foulant accumulation $\left(g m^{2} L^{-2}\right)$ & $10^{9}$ \\
$\hat{K}$ & Foulant removal $\left(m^{2} L^{-1}\right)$ & variable \\
$c$ & Reciprocal of Foulant Removal, no EPS. $\left(m^{-2} L\right)$ & 100 \\
$m$ & EPS production rate $\left(S^{-2}\right)$ & 10 \\
$\delta$ & EPS decay rate $\left(S^{-2}\right)$ & $10^{-5}$ \\
$\alpha$ & $\hat{K}$ decay from EPS $m^{2} L^{-1}$ & $10^{6}$ \\
\hline
\end{tabular}

Table 1: Description of variables and parameters used in this study. The parameters are similar to those used in our previous study. The parameter $c$ is used so that $\hat{K}$ for a clean filter is the same as in (28). All the qualitative statements are carried over from (28) for broad ranges of parameters. 
optimal, the backwashing needs to be performed quickly, which is consistent with various observations regarding the rate of cycling $(41,42)$.

\section{Conclusions}

The aim of this study was two-fold. First, we show that our optimal control methodology can be adapted to more complicated systems, where analytic methods fail. Second, we can show quantitive differences between the predicted optimal control strategies and the subsequent recovered volume. For the first aspect, the main difference is the numerical solutions of the state and adjoint equations rather than analytic solutions to the ODE's. Any methodology that provides an approximate of the time-course will suffice. For the second, we see that as the various aspects of fouling are considered, the total volume that can be filtered decreases. The fouling is determined primarily by the accumulation on the membrane. This is relatively expected - what is not expected is how the anticipate the change in the control strategy.

Our quantitative estimates indicate that it is the fouling of the filter by EPS rather than the irreversibility of the attachment that leads the decline in flux. Additionally, the change in control strategy is not as straightforward as one might have supposed. In particular, intuitively one might expect that to control the EPS fouling, we should reverse the flow earlier. Here we find the reverse. It is possible that a more detailed model of EPS dynamics (including considering EPS production by bacteria attached to membrane surfaces) needs to be considered - and that is one direction we aim to proceed.

Additionally, it is known that EPS is not the only constituent responsible for irreversible attachment. Natural organic matter (NOM) and cake development play a significant role in flux decline as well as irreversible attachment $(43,44)$. The methodology described here can be extended to address this by including more detailed modeling of both the attachment process since NOM attachment is heavily dependent on the hydrophobicity, solution chemistry and feed water properties. We have also simplified the biofilm/gel/cake dynamics - and these play a significant role in the flux decline as well as irreversibility. In general, however, we have developed a flexible method for implementing a wide variety of physical and biological processes while still maintaining a predictive framework. 


\section{Acknowledgements}

NC was supported by NSF CBET 1510743. SC was supported by NSF CBET 1636104 .

\section{References}

[1] S. Chellam. Microfiltration. In Eric Michael Vrijen Hoek and Volodymyr V Tarabara, editors, Encyclopedia of Membrane Science and Technology, chapter Microfiltration, pages 1-21. John Wiley \& Sons, New Jersey, 2013.

[2] J. Kilduff. Ultrafiltration. In Eric Michael Vrijen Hoek and Volodymyr V Tarabara, editors, Encyclopedia of Membrane Science and Technology, chapter Ultrafiltration, pages 1-34. John Wiley \& Sons, New Jersey, 2013.

[3] Yuanqing Chao and Tong Zhang. Growth behaviors of bacteria in biofouling cake layer in a dead-end microfiltration system. Bioresource Technology, 102(2):1549-1555, 2011.

[4] R. Ghosh. Applications of membranes in biotechnology. In Eric Michael Vrijen Hoek and Volodymyr V Tarabara, editors, Encyclopedia of Membrane Science and Technology, chapter Application of Membranes in Biotechnology, pages 1-19. John Wiley \& Sons, New Jersey, 2013.

[5] W. Xu and S. Chellam. Initial stages of bacterial fouling of microfiltration membranes. Environmental Science and Technology, 39(17):64706476, 2005.

[6] Shankararaman Chellam, Joseph G Jacangelo, and Thomas P Bonacquisti. Modeling and experimental verification of pilot-scale hollow fiber, direct flow microfiltration with periodic backwashing. Environmental Science and Technology, 32(1):75-81, 1998.

[7] Ebrahim Akhondi, Filicia Wicaksana, and Anthony Gordon Fane. Evaluation of fouling deposition, fouling reversibility and energy consumption of submerged hollow fiber membrane systems with periodic backwash. Journal of Membrane Science, 452:319-331, 2014. 
[8] EP Jacobs, SM Bradshaw, JP Botes, and VL Pillay. Reverse-pressure back-flush in pilot scale, dead-end ultrafiltration of surface water. Journal of Membrane Science, 252(1):51-63, 2005.

[9] Wayne F Jones, Richard L Valentine, and VGJ Rodgers. Removal of suspended clay from water using transmembrane pressure pulsed microfiltration. Journal of membrane science, 157(2):199-210, 1999.

[10] Wendy D Mores, Christopher N Bowman, and Robert H Davis. Theoretical and experimental flux maximization by optimization of backpulsing. Journal of Membrane Science, 165(2):225-236, 2000.

[11] Sanjeev Redkar, Vinod Kuberkar, and Robert H Davis. Modeling of concentration polarization and depolarization with high-frequency backpulsing. Journal of Membrane Science, 121(2):229-242, 1996.

[12] PJ Remize, C Guigui, and C Cabassud. Evaluation of backwash efficiency, definition of remaining fouling and characterisation of its contribution in irreversible fouling: Case of drinking water production by air-assisted ultra-filtration. Journal of Membrane Science, 355(1):104111, 2010.

[13] Hiroshi Yamamura, Katsuki Kimura, Takaharu Okajima, Hiroshi Tokumoto, and Yoshimasa Watanabe. Affinity of functional groups for membrane surfaces: implications for physically irreversible fouling. Environmental science 83 technology, 42(14):5310-5315, 2008.

[14] M Raffin, E Germain, and SJ Judd. Influence of backwashing, flux and temperature on microfiltration for wastewater reuse. Separation and purification technology, 96:147-153, 2012.

[15] Haiqing Chang, Fangshu Qu, Baicang Liu, Huarong Yu, Kai Li, Senlin Shao, Guibai Li, and Heng Liang. Hydraulic irreversibility of ultrafiltration membrane fouling by humic acid: Effects of membrane properties and backwash water composition. Journal of Membrane Science, 493:723-733, 2015.

[16] Appala Raju Badireddy, Shankararaman Chellam, Svetlana Yanina, Paul Gassman, and Kevin M Rosso. Bismuth dimercaptopropanol (bisbal) inhibits the expression of extracellular polysaccharides and proteins 
by Brevundimonas diminuta: implications for membrane microfiltration. Biotechnology and Bioengineering, 99(3):634-643, 2008.

[17] Shankararaman Chellam and Wendong Xu. Blocking laws analysis of dead-end constant flux microfiltration of compressible cakes. Journal of Colloid and Interface Science, 301(1):248-257, 2006.

[18] Hongjun Lin, Meijia Zhang, Fangyuan Wang, Fangang Meng, BaoQiang Liao, Huachang Hong, Jianrong Chen, and Weijue Gao. A critical review of extracellular polymeric substances (EPSs) in membrane bioreactors: characteristics, roles in membrane fouling and control strategies. Journal of Membrane Science, 460:110-125, 2014.

[19] Anja Drews, Chung-Hak Lee, and Matthias Kraume. Membrane foulinga review on the role of EPS. Desalination, 200(1):186-188, 2006.

[20] Y Ye, P Le Clech, V Chen, and AG Fane. Evolution of fouling during crossflow filtration of model EPS solutions. Journal of Membrane Science, 264(1):190-199, 2005.

[21] K Katsoufidou, SG Yiantsios, and AJ Karabelas. Experimental study of ultrafiltration membrane fouling by sodium alginate and flux recovery by backwashing. Journal of Membrane Science, 300(1):137-146, 2007.

[22] Alison E Contreras, Albert Kim, and Qilin Li. Combined fouling of nanofiltration membranes: mechanisms and effect of organic matter. Journal of Membrane Science, 327(1):87-95, 2009.

[23] Moshe Herzberg, Seoktae Kang, and Menachem Elimelech. Role of extracellular polymeric substances (eps) in biofouling of reverse osmosis membranes. Environmental science \&3 technology, 43(12):4393-4398, 2009.

[24] Jost Wingender, Thomas R Neu, and Hans-Curt Flemming. Microbial extracellular polymeric substances: characterization, structure and function. Springer Science \& Business Media, 2012.

[25] R Khan, F Shen, K Khan, LX Liu, HH Wu, JQ Luo, and YH Wan. Biofouling control in a membrane filtration system by a newly isolated novel quorum quenching bacterium, Bacillus methylotrophicus sp. wy. RSC Advances, 6(34):28895-28903, 2016. 
[26] Zbigniew Lewandowski and Haluk Beyenal. Fundamentals of biofilm research. CRC press, 2013.

[27] Shankararaman Chellam and NG Cogan. Colloidal and bacterial fouling during constant flux microfiltration: Comparison of classical blocking laws with a unified model combining pore blocking and eps secretion. Journal of Membrane Science, 382(1):148-157, 2011.

[28] NG Cogan and Shankararaman Chellam. A method for determining the optimal back-washing frequency and duration for dead-end microfiltration. Journal of Membrane Science, 469:410-417, 2014.

[29] Georges Belfort, Robert H Davis, and Andrew L Zydney. The behavior of suspensions and macromolecular solutions in crossflow microfiltration. Journal of Membrane Science, 96(1-2):1-58, 1994.

[30] WR Bowen, JI Calvo, and A Hernandez. Steps of membrane blocking in flux decline during protein microfiltration. Journal of Membrane Science, 101(1):153-165, 1995.

[31] J. Hermia. Constant pressure blocking filtration laws - application to power-law non-newtonian fluids. Transactions of the Institution of Chemical Engineers, 60:182-187, 1982.

[32] NG Cogan and Shankar Chellam. Incorporating pore blocking, cake filtration, and EPS production in a model for constant pressure bacterial fouling during dead-end microfiltration. Journal of Membrane Science, 345(1):81-89, 2009.

[33] Chia-Chi Ho and Andrew L. Zydney. A combined pore blockage and cake filtration model for protein fouling during microfiltration. Journal of Colloid and Interface Science, 232:389-399, 2000.

[34] R Kommedal, R Bakke, J Dockery, and P Stoodley. Modelling production of extracellular polymeric substances in a Pseudomonas aeruginosa chemostat culture. Water Science and Technology, 43(6):129-134, 2001.

[35] Erik Alpkvista and Isaac Klapper. A multidimensional multispecies continuum model for heterogeneous biofilm development. Bulletin of Mathematical Biology, 69(2):765-789, 2007. 
[36] Munir Cheryan. Ultrafiltration and microfiltration handbook. CRC press, 1998.

[37] Anselm Omoike and Jon Chorover. Spectroscopic study of extracellular polymeric substances from Bacillus subtilis: aqueous chemistry and adsorption effects. Biomacromolecules, 5(4):1219-1230, 2004.

[38] I Horcas, Rs Fernández, JM Gomez-Rodriguez, J Colchero, JWSXM Gómez-Herrero, and AM Baro. Wsxm: a software for scanning probe microscopy and a tool for nanotechnology. Review of Scientific Instruments, 78(1):013705, 2007.

[39] Leah Edelstein-Keshet. Mathematical models in biology, volume 46. Siam, 1988.

[40] Suzanne M Lenhart and John T Workman. Optimal control applied to biological models, volume 15. CRC Press, 2007.

[41] Charles S Parnham and Robert H Davis. Protein recovery from bacterial cell debris using crossflow microfiltration with backpulsing. Journal of membrane science, 118(2):259-268, 1996.

[42] Caroline Wilharm and VGJ Rodgers. Significance of duration and amplitude in transmembrane pressure pulsed ultrafiltration of binary protein mixtures. Journal of Membrane Science, 121(2):217-228, 1996.

[43] Linhua Fan, John L Harris, Felicity A Roddick, and Nic A Booker. Influence of the characteristics of natural organic matter on the fouling of microfiltration membranes. Water Research, 35(18):4455-4463, 2001.

[44] Catherine Jucker and Mark M Clark. Adsorption of aquatic humic substances on hydrophobic ultrafiltration membranes. Journal of Membrane Science, 97:37-52, 1994. 


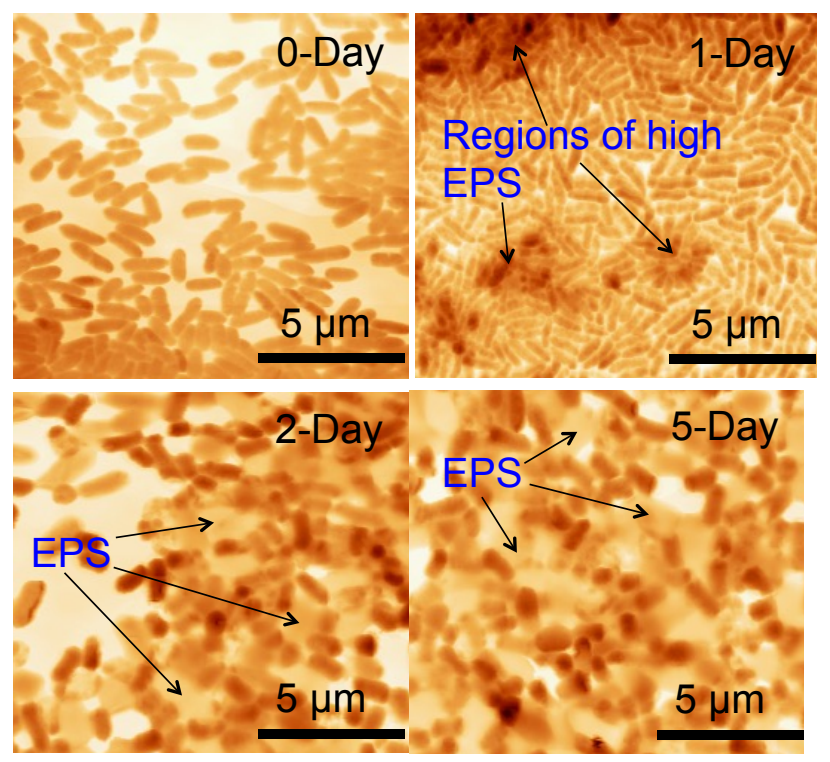

Figure 1: Atomic force microscopy provided visual confirmation of EPS expression. The beginnings of a thin slime-like EPS layer can be seen after 1-day, which increased substantially for the 2-day and 5-day samples. These micrographs corroborate increasing EPS secretion measured colorimetrically and also provide qualitative evidence that $B$. diminuta cells remained intact (insignificant lysis) over the duration of the experiments indicating that intracellular material did not contaminate EPS polysaccharides and proteins measurements. 


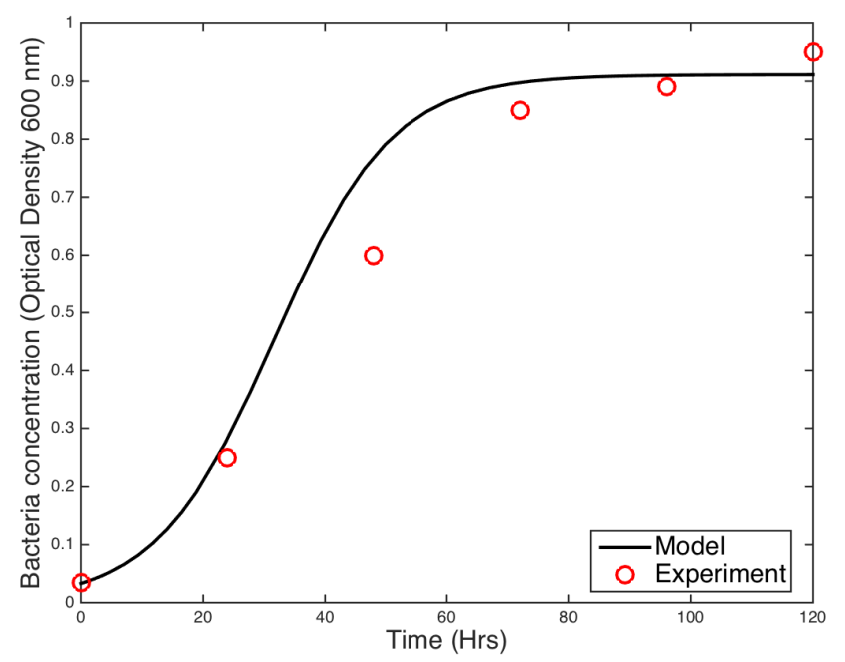

(a)

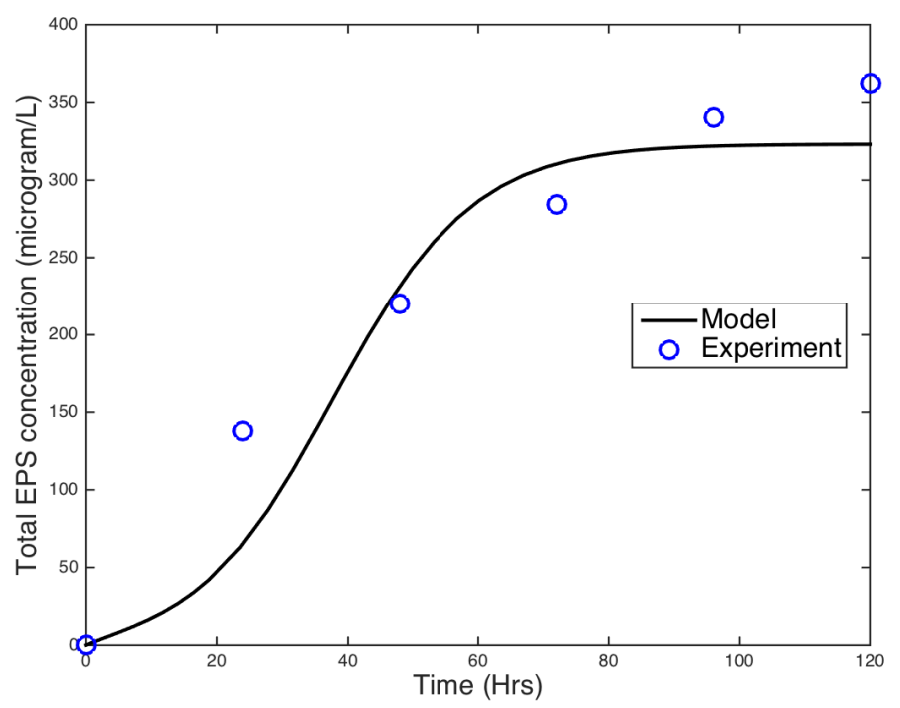

(b)

Figure 2: Comparison between experiment and simulation of bacterial growth (a) and EPS accumulation (b). We use this to determine an estimate of the production and decay kinetics of EPS for our filtration model. 


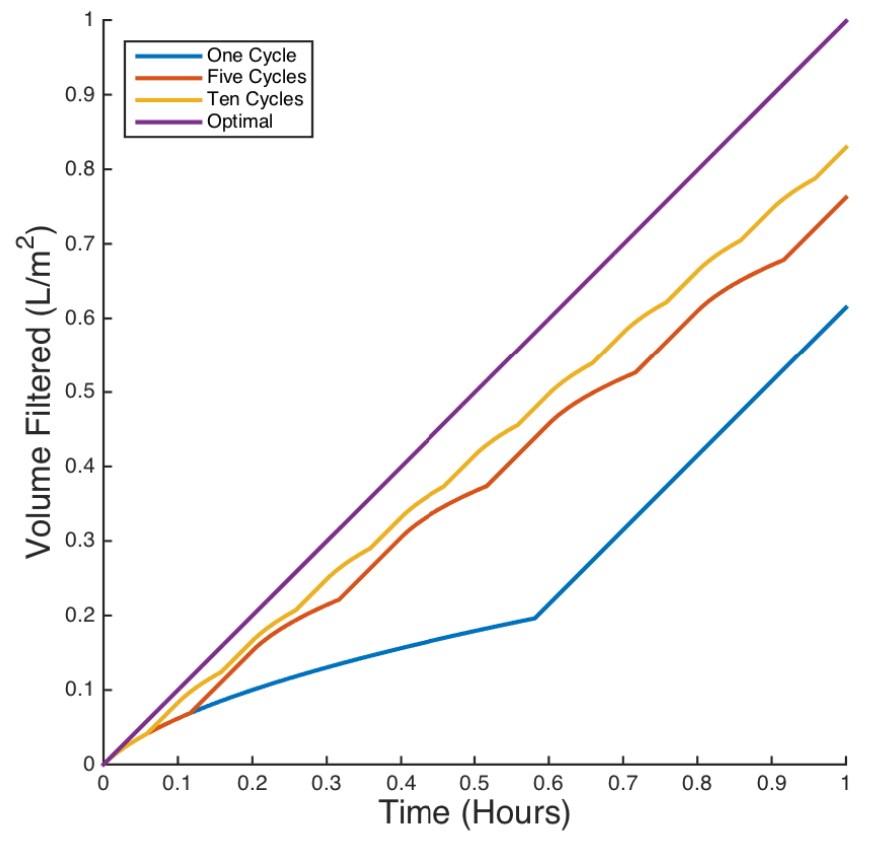

Figure 3: We show the total volume filtered (where we have scaled the flux and assumed a unit membrane area) as a function of time. This is the base case where EPS does not play a role in the fouling (e.g. $\mu_{2}$ and $\alpha$ are zero). 


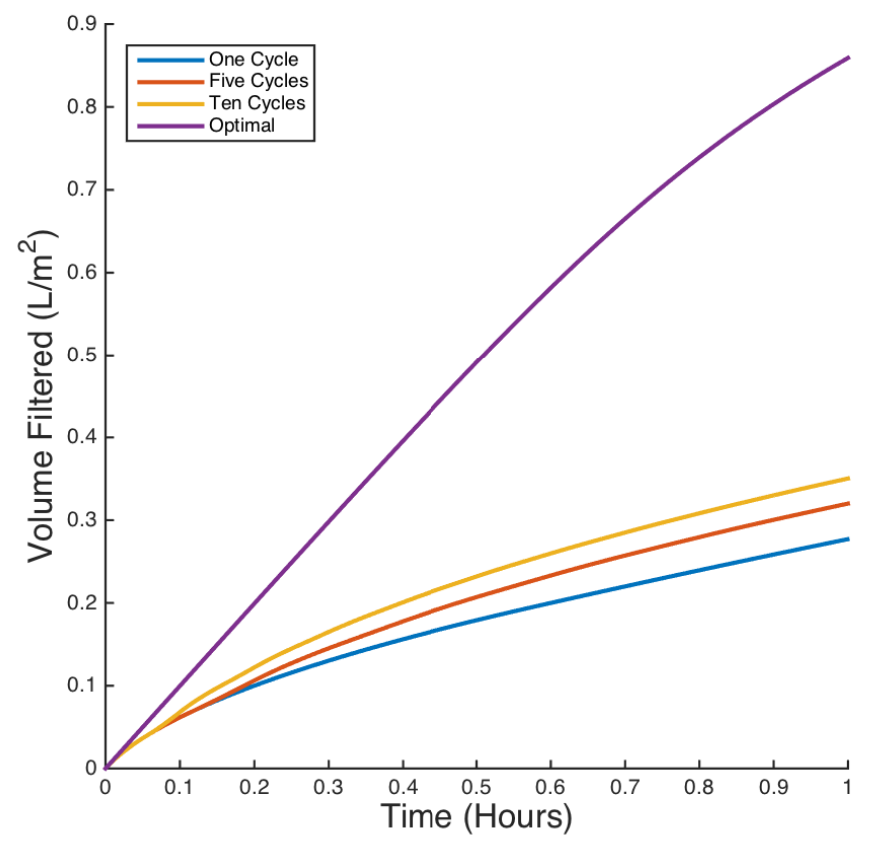

Figure 4: We show the total volume filtered (where we have scaled the flux and assumed a unit membrane area) as a function of time. In this case EPS does not play a role in reducing the flux, but does inhibit removal (e.g. $\left.\mu_{2}=0\right)$. 


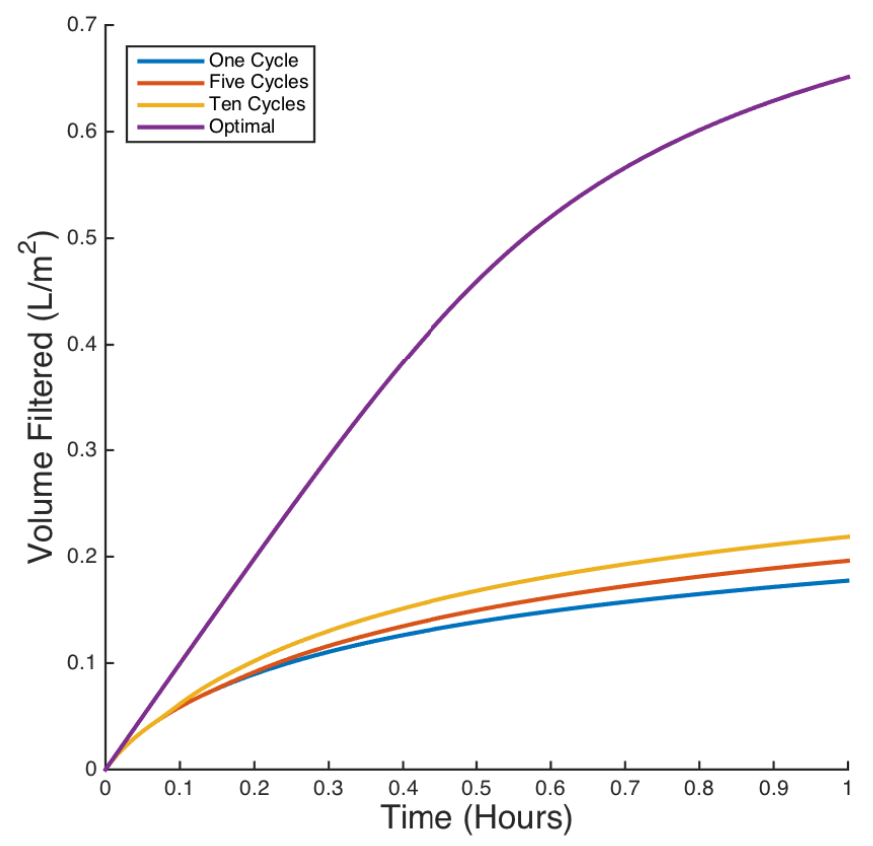

Figure 5: We show the total volume filtered (where we have scaled the flux and assumed a unit membrane area) as a function of time. In this case EPS reduces the flux and inhibits removal (e.g. $\mu_{2} \neq 0$ and $\alpha \neq 0$ ). 